\title{
Biogenic Amine Metabolic Process
}

National Cancer Institute

\section{Source}

National Cancer Institute. Biogenic Amine Metabolic Process. NCI Thesaurus. Code C40625.

Biogenic Amine Metabolism involves anabolic and catabolic biochemical changes to a type of nitrogen containing hydrocarbons (biogenic amines) involved in signaling as neurotransmitters or hormones or as components of vitamins, phospholipids, or ribosomes within a cell as materials needed for important life processes. 